\title{
Higher Order Deformations of Complex Structures ${ }^{\star}$
}

\author{
Eric D'HOKER † and Duong H. PHONG ${ }^{\ddagger}$ \\ $\dagger$ Department of Physics and Astronomy, University of California, Los Angeles 90024, USA \\ E-mail: dhoker@physics.ucla.edu \\ $\ddagger$ Department of Mathematics, Columbia University, New York, NY 10027, USA \\ E-mail: phong@math.columbia.edu
}

Received March 27, 2015, in final form June 15, 2015; Published online June 23, 2015

http://dx.doi.org/10.3842/SIGMA.2015.047

\begin{abstract}
Deformations of complex structures by finite Beltrami differentials are considered on general Riemann surfaces. Exact formulas to any fixed order are derived for the corresponding deformations of the period matrix, Green's functions, and correlation functions in conformal field theories with vanishing total central charge. The stress tensor is shown to give a simple representation of these deformations valid to all orders. Such deformation formulas naturally enter into the evaluation of superstring amplitudes at twoloop order with Ramond punctures, and at higher loop order, in the supergravity formulation of the RNS superstring.
\end{abstract}

Key words: Beltrami differentials; deformations of covariant derivatives; stress tensor; conformal invariance

2010 Mathematics Subject Classification: 32G05; 51M15; 51P05; 53Z05

\section{Introduction}

It is a fundamental property of the moduli space of Riemann surfaces that its tangent space is given by the space of Beltrami differentials, modulo the range of the $\bar{\partial}$ operator on vector fields. In general, without some additional structure, such as a connection, a tangent vector to a manifold determines only an infinitesimal deformation on the manifold. However, in the case of moduli space, we can actually associate to each Beltrami differential a finite deformation of the underlying complex structure. It is the purpose of this note to work out in detail the properties of this finite deformation, including explicit formulas to all orders in the Beltrami differential for the resulting period matrix and Green functions. Similarly, it is well-known that infinitesimally, the deformations of the correlation functions of a conformal field theory can be obtained by a single insertion of the stress tensor. We show that a simple exponentiated generalization of this formula remains valid to all orders in a finite deformation, assuming the vanishing of the total central charge.

The problem of higher order deformations of complex structures arises in superstring perturbation theory. There the period matrix of a super Riemann surface can receive corrections of higher order as the gravitino field is turned on. For the scattering of Neveu-Schwarz states in genus 2, deformations by a Beltrami differential are needed only to first order [6, 7, 9] (see also [10] for a review). This is no longer the case for genus 3 or higher, or even for genus 2 , when the scattering of Ramond states is considered $[2,18]$. The results of the present note will play, in particular, an essential role in the construction of string amplitudes with Ramond states at two-loop level. We shall report on these issues elsewhere.

\footnotetext{
${ }^{\star}$ This paper is a contribution to the Special Issue on Exact Solvability and Symmetry Avatars in honour of Luc Vinet. The full collection is available at http://www.emis.de/journals/SIGMA/ESSA2014.html
} 


\section{Finite deformations by Beltrami differentials}

Let $\Sigma$ be a compact Riemann surface, and let $z$ denote a local holomorphic coordinate on the surface. Let $\mu=\mu(z) d \bar{z} \otimes \partial_{z}$ be a Beltrami differential. We shall always assume that the reduced part $\mu_{\text {red }}$ of $\mu$ satisfies $\left|\mu_{\text {red }}\right|<1$, and that $\mu$ has even grading in a finitely-generated Grassmann algebra. This last situation is the one of interest in superstring perturbation theory, where furthermore $\mu$ is actually a nilpotent element of finite order of a Grassmann algebra, the order being related to the number of odd moduli in the problem.

We define a new, deformed, complex structure on $\Sigma$ by the requirement that its local holomorphic coordinate $w$ satisfy the equation

$$
\left(\partial_{\bar{z}}+\mu(z) \partial_{z}\right) w=0 .
$$

The well-known theorem of Beltrami asserts that a local holomorphic coordinate $w$ always exists, and that the partial derivative $\partial_{z} w$ may be taken to be non-vanishing. We shall often distinguish the two complex structures by indicating their respective local complex coordinate, either $z$ for the original complex structure or $w$ for the deformed complex structure. For a given complex structure, we decompose the cotangent bundle to $\Sigma$ into the canonical bundle $K$ and its complex conjugate $\bar{K}$, whose sections are referred to as forms of type $(1,0)$ and $(0,1)$ respectively. When several complex structures, say $z$ and $w$, are simultaneously at play we shall use the notations $K_{z}$ and $K_{w}$ for their respective cotangent bundles.

\subsection{Deformation of holomorphic 1-forms}

Consider now a holomorphic (1,0)-form $\omega$ with respect to the deformed complex structure $w$, so that $\omega$ is a holomorphic section of $K_{w}$, and takes the form $\omega=\omega(w) d w$. The basic observation is that, expressed in terms of $z$ coordinates, a holomorphic $(1,0)$-form with respect to the complex structure $w$ must be of the form

$$
\omega=\varphi d z-(\mu \varphi) d \bar{z}
$$

with the coefficient function $\varphi$ satisfying the relation

$$
\partial_{\bar{z}} \varphi=-\partial_{z}(\mu \varphi) .
$$

Indeed, since we have the equations

$$
\begin{aligned}
& d w=\left(\partial_{z} w\right) d z+\left(\partial_{\bar{z}} w\right) d \bar{z}=\left(\partial_{z} w\right)(d z-\mu(z) d \bar{z}), \\
& \partial_{\bar{w}}=\left(\partial_{\bar{w}} z\right) \partial_{z}+\left(\partial_{\bar{w}} \bar{z}\right) \partial_{\bar{z}}=\left(\partial_{\bar{w}} \bar{z}\right)\left(\partial_{\bar{z}}+\mu(z) \partial_{z}\right),
\end{aligned}
$$

it follows that $\omega$ can be expressed in the form (2.1) by taking $\varphi=\left(\partial_{z} w\right) \omega(w)$. Furthermore, the holomorphicity of a $(1,0)$-form is equivalent to the fact that it is closed, which can be implemented using either set of coordinates, $z$ or $w$. Using the coordinate $z$, we find that $d \omega=0$ is equivalent (2.2), giving the desired equality.

The general solution of equation (2.2) is given by the following integral equation ${ }^{1}$

$$
\varphi(z)=\psi(z)+\frac{1}{2 \pi} \int_{\Sigma} d^{2} z^{\prime} \partial_{z} \partial_{z^{\prime}} \ln E\left(z, z^{\prime}\right)(\mu \varphi)\left(z^{\prime}\right),
$$

where $\psi=\psi(z) d z$ is an arbitrary holomorphic $(1,0)$-form and $E\left(z, z^{\prime}\right)$ is the prime form, both with respect to the complex structure $z$. Successively iterating (2.4) gives an expression for $\varphi$ to an arbitrary order in $\mu$. This iteration process terminates after a finite number of iterations to give the exact solution when $\mu$ is a nilpotent element valued in a finitely-generated Grassmann algebra, which is the case in superstring perturbation theory.

\footnotetext{
${ }^{1}$ Throughout, we shall use the conventions of [4], such as the normalization $d^{2} z=i d z \wedge d \bar{z}$.
} 


\subsection{Deformation of the period matrix}

We can evaluate now the deformation of the period matrix of the Riemann surface $\Sigma$ to arbitrarily high order in $\mu$. For this, we fix a canonical homology basis $A_{I}, B_{I}, 1 \leq I \leq h$, \# $\left(A_{I} \cap B_{J}\right)=\delta_{I J}$, $\#\left(A_{I} \cap A_{J}\right)=0$, \# $\left(B_{I} \cap B_{J}\right)=0$, where $h$ is the genus of $\Sigma$. Let $\psi_{I}(z) d z$ be the basis of holomorphic 1-forms with respect to the complex structure $z$ which is dual to the cycles $A_{I}$, and let $\Omega_{I J}$ be the matrix of their $B_{J}$ periods

$$
\oint_{A_{J}} \psi_{I}(z) d z=\delta_{I J}, \quad \oint_{B_{J}} \psi_{I}(z) d z=\Omega_{I J}
$$

Corresponding to the basis $\psi_{I}$ is a basis $\omega_{I}$ of holomorphic $(1,0)$-forms with respect to the $w$ structure, with $\omega_{I}=\varphi_{I}(z) d z-\left(\mu \varphi_{I}\right)(z) d \bar{z}$ and $\varphi_{I}(z)$ given by $(2.4)$

$$
\varphi_{I}(z)=\psi_{I}(z)+\frac{1}{2 \pi} \int_{\Sigma} d^{2} z^{\prime} \partial_{z} \partial_{z^{\prime}} \ln E\left(z, z^{\prime}\right)\left(\mu \varphi_{I}\right)\left(z^{\prime}\right)
$$

Their periods around a closed cycle $C$ are given by

$$
\oint_{C} \omega_{I}(w) d w=\oint_{C}\left(\varphi_{I}(z) d z-\left(\mu \varphi_{I}\right)(z) d \bar{z}\right) .
$$

To express these periods in terms of the complex structure $z$ and the Beltrami differential $\mu$, we substitute in this formula the right hand side of (2.5) for $\varphi_{I}$. But due to the fact that the integral of $\partial_{z} \partial_{z^{\prime}} \ln E\left(z, z^{\prime}\right)$ is only conditionally convergent, the interchange of the order of integrations in $z$ and $z^{\prime}$ is found to require a correction term, and is given by the following formula proved in $[5]$

$$
\begin{aligned}
& \frac{1}{2 \pi} \oint_{C} d z\left(\int_{\Sigma} d^{2} z^{\prime} \partial_{z} \partial_{z^{\prime}} \ln E\left(z, z^{\prime}\right)\left(\mu \varphi_{I}\right)\left(z^{\prime}\right)\right) \\
& \quad=\frac{1}{2 \pi} \int_{\Sigma} d^{2} z^{\prime}\left(\oint_{C} d z \partial_{z} \partial_{z^{\prime}} \ln E\left(z, z^{\prime}\right)\right)\left(\mu \varphi_{I}\right)\left(z^{\prime}\right)+\oint_{C}\left(\mu \varphi_{I}\right)(z) d \bar{z} .
\end{aligned}
$$

Combining (2.6) and (2.7), we see that the integral over the $(0,1)$-form $\left(\mu \varphi_{I}\right) d \bar{z}$ cancels out, and we are left with

$$
\oint_{C} \omega_{I}(w) d w=\oint_{C} \psi_{I}(z) d z+\frac{1}{2 \pi} \int_{\Sigma} d^{2} z^{\prime}\left(\oint_{C} \partial_{z} \partial_{z^{\prime}} \ln E\left(z, z^{\prime}\right) d z\right)\left(\mu \varphi_{I}\right)\left(z^{\prime}\right) .
$$

Now the monodromy of the prime form $E(z, w)$ is trivial around $A_{I^{-c y c l e s}}$, and around $B_{I^{-}}$cycles is given by

$$
E\left(z+B_{J}, z^{\prime}\right)=E\left(z, z^{\prime}\right) \exp \left(-\pi i \Omega_{J J}+2 \pi i \int_{z}^{z^{\prime}} \omega_{J}\right) .
$$

Letting $C=A_{J}$ or $C=B_{J}$ in the preceding formula, we find

$$
\oint_{A_{J}} \omega_{I}(w) d w=\delta_{I J}, \quad \oint_{B_{J}} \omega_{I}(w) d w=\Omega_{I J}+i \int_{\Sigma} d^{2} z \omega_{J}(z) \mu(z) \varphi_{I}(z)
$$

where $\varphi_{I}\left(z^{\prime}\right)$ may be obtained by successive iterations of $(2.4)$.

Thus, the forms $\omega_{I}$ precisely provide the basis of holomorphic one-forms with respect to the deformed complex structure $w$ which is dual to the cycles $A_{I}$. Their $B_{J}$-periods provide the period matrix $\Omega_{I J}(\mu)$ of the complex structure $w$ in terms of the period matrix $\Omega_{I J}=\Omega_{I J}(0)$ of 
the original complex structure $z$. Repeated iteration of the formula $(2.5)$ for $\varphi_{I}$ produces $\Omega_{I J}(\mu)$ to an arbitrary fixed order in a power expansion of $\mu$. For example, to order $\mathcal{O}\left(\mu^{3}\right)$, we obtain

$$
\begin{aligned}
\Omega_{I J}(\mu)= & \Omega_{I J}+i \int_{\Sigma} d^{2} z \omega_{J}(z) \mu(z) \omega_{I}(z) \\
& +\frac{i}{2 \pi} \int_{\Sigma} d^{2} z \int_{\Sigma} d^{2} z^{\prime} \omega_{J}(z) \mu(z) \partial_{z} \partial_{z^{\prime}} \ln E\left(z, z^{\prime}\right) \mu\left(z^{\prime}\right) \omega_{I}\left(z^{\prime}\right)+\mathcal{O}\left(\mu^{3}\right) .
\end{aligned}
$$

For fixed $z$, the integral over $z^{\prime}$ is conditionally convergent, but may naturally be defined uniquely by requiring that the integral over $z^{\prime}$ of the singularity $\left(z-z^{\prime}\right)^{-2}$ over a small circular disc centered at $z$ must vanish by angular integration.

\section{Deformations of tensors and covariant derivatives}

Next, we shall consider deformations of tensors of more general weight. It will be convenient to denote by $T^{m, n}\left(\Sigma_{z}\right)$ the space of sections of $K_{z}^{\otimes m} \otimes \bar{K}_{z}^{\otimes n}$, namely the space of tensors of type $\varphi(z)(d z)^{m} \otimes(d \bar{z})^{n}$, where $K_{z}$ is the canonical bundle for complex structure $z$. Here, the subindex $z$ continues to indicate the complex structure. Spinors may be included as well by taking half-integer $m$ and $n$, and providing the additional data of a spin structure.

\subsection{Deformation of tensors}

Now consider a tensor $\varphi(z)(d z)^{n}$ belonging to $T^{(n, 0)}\left(\Sigma_{z}\right)$ for given $n$. Then the correspondence $\omega d w \leftrightarrow \varphi d z$ used in the previous section for the special case $n=1$ generalizes to the following correspondences for tensors of type $\varphi(z)(d z)^{n}$

$$
\begin{aligned}
& \iota_{w \leftarrow z}: \varphi(z) \rightarrow \tilde{\varphi}(w)=\varphi(z)\left(\partial_{w} z\right)^{n}, \\
& \iota_{z \leftarrow w}: \varphi(w) \rightarrow \tilde{\varphi}(z)=\varphi(w)\left(\partial_{z} w\right)^{n} .
\end{aligned}
$$

Intrinsically, the map $\iota_{z \leftarrow w}$ is proportional to the projection from the space $T^{n, 0}\left(\Sigma_{w}\right)$ onto the space $T^{n, 0}\left(\Sigma_{z}\right)$, defined by the direct sum decomposition

$$
T^{n, 0}\left(\Sigma_{w}\right)=\bigoplus_{k=0}^{n} T^{n-k, k}\left(\Sigma_{z}\right)
$$

and keeping only the top component $T^{n, 0}\left(\Sigma_{z}\right)$. This decomposition may be carried out explicitly, with the help of $(2.3)$

$$
\varphi(w)(d w)^{n}=\sum_{k=0}^{n} \frac{n !}{k !(n-k) !}(-\mu)^{k} \varphi(w)\left(\partial_{z} w\right)^{n}(d z)^{n-k} \otimes(d \bar{z})^{k} .
$$

The map $\iota_{z \leftarrow w}$ corresponds to retaining only the term $k=0$. Similarly, the inverse map $\iota_{w \leftarrow z}$ is defined by the analogous projection from $T^{n, 0}\left(\Sigma_{z}\right)$ onto $T^{n, 0}\left(\Sigma_{w}\right)$. We note that the maps $\iota_{z \leftarrow w}$ and $\iota_{w \leftarrow z}$ restricted to the spaces $T^{n, 0}\left(\Sigma_{w}\right)$ onto $T^{n, 0}\left(\Sigma_{z}\right)$ are not inverses of each other. A simple calculation gives their composition as follows

$$
\iota_{z \leftarrow w} \iota_{w \leftarrow z}=\left(\partial_{z} w\right)^{n}\left(\partial_{w} z\right)^{n}=\frac{1}{(1-\mu \bar{\mu})^{n}} .
$$

Thus, the maps are not properly projections but are proportional to projections. 


\subsection{Deformation of covariant derivatives}

In the treatment of deformations of one-forms in the previous section, it sufficed to use the de Rham exterior derivative. However, for tensors of higher rank we shall need covariant derivatives. So we introduce now deformations of metrics associated to deformations of complex structures. Let $\Sigma_{z}$ be a Riemann surface with local holomorphic coordinates $z$, and let $\mu$ be a Beltrami differential which deforms the complex structure $z$ to $w$, for the Riemann surface $\Sigma_{w}$. Let the metrics corresponding to $\Sigma_{z}$ and $\Sigma_{w}$ be denoted respectively by $d s^{2}=2 g_{\bar{z} z} d z d \bar{z}$ and $d \tilde{s}^{2}=$ $2 \tilde{g}_{\bar{w} w} d w d \bar{w}$. The covariant derivatives $\nabla^{z}$ on a form $\varphi=\varphi(z)(d z)^{n}$ in $T^{n, 0}\left(\Sigma_{z}\right)$ and $\tilde{\nabla}^{w}$ on a form $\tilde{\varphi}=\tilde{\varphi}(w)(d w)^{n}$ in $T^{n, 0}\left(\Sigma_{w}\right)$ are defined as usual by relations which do not require a connection

$$
\nabla^{z} \varphi=g^{z \bar{z}} \partial_{\bar{z}} \varphi, \quad \tilde{\nabla}^{w} \tilde{\varphi}=\tilde{g}^{w \bar{w}} \partial_{\bar{w}} \tilde{\varphi} .
$$

We now define the deformation $\tilde{\nabla}^{z}$ of the covariant derivative $\nabla^{z}$ by the Beltrami differential $\mu$ to be the following operator acting on a form $\varphi(z)(d z)^{n}$ in $T^{n, 0}\left(\Sigma_{z}\right)$

$$
\tilde{\nabla}^{z} \varphi=\iota_{w \leftarrow z}^{-1}\left(\tilde{\nabla}^{w}\left(\iota_{w \leftarrow z} \varphi\right)\right) .
$$

The following surprisingly simple and exact formula for $\tilde{\nabla}^{z}$ holds to all orders in $\mu$. With the help of the Weyl factor $e^{2 \sigma}=\left|\partial_{z} w\right|^{2} g^{z \bar{z}} \tilde{g}_{w \bar{w}}$ between the metrics, one finds

$$
\tilde{\nabla}^{z} \varphi=\frac{e^{-2 \sigma} g^{z \bar{z}}}{(1-\mu \bar{\mu})^{2}}\left(\partial_{\bar{z}} \varphi+\mu \partial_{z} \varphi+n \varphi \partial_{z} \mu-n \varphi\left(\partial_{\bar{z}}+\mu \partial_{z}\right) \ln (1-\mu \bar{\mu})\right),
$$

where $\mu, \bar{\mu}$, and $\varphi$ are functions of $z$. To the best of the authors' knowledge, this formula is new. It is a generalization to all orders in $\mu$, namely for a finite deformation of complex structures, of the well-known formulas of Friedan [11] for the case of infinitesimal $\mu$. Finite deformations of Abelian differentials on Riemann surfaces have also been considered in the mathematical literature, in particular in $[14,19]$.

To establish (3.2), we start from the defining formula for $\tilde{\nabla}^{z}$

$$
\tilde{\nabla}^{z} \varphi=\left(\partial_{w} z\right)^{-(n-1)} \tilde{g}^{w \bar{w}} \partial_{\bar{w}}\left(\varphi(z)\left(\partial_{w} z\right)^{n}\right) .
$$

Expressing $\partial_{\bar{w}}$ in terms of derivatives with respect to $z$ and $\bar{z}$ using the second line in (2.3), and eliminating the metric $\tilde{g}^{w \bar{w}}$ using the definition of the Weyl factor, we find

$$
\tilde{\nabla}^{z} \varphi=\frac{e^{-2 \sigma} g^{z \bar{z}}}{(1-\mu \bar{\mu})^{2}}\left(\left(\partial_{\bar{z}}+\mu(z) \partial_{z}\right) \varphi(z)+n \varphi(z)\left(\partial_{\bar{z}}+\mu(z) \partial_{z}\right) \ln \left(\partial_{w} z\right)\right) .
$$

Since all functions are now with respect to $z$, we shall no longer exhibit this dependence below. Using (3.1) to eliminate $\ln \left(\partial_{w} z\right)$ in terms of $\ln \left(\partial_{z} w\right)$ and $\ln (1-\mu \bar{\mu})$, we find

$$
\tilde{\nabla}^{z} \varphi=\frac{e^{-2 \sigma} g^{z \bar{z}}}{(1-\mu \bar{\mu})^{2}}\left(\left(\partial_{\bar{z}}+\mu \partial_{z}\right) \varphi-n \varphi\left(\partial_{\bar{z}}+\mu \partial_{z}\right)\left\{\ln \left(\partial_{z} w\right)+\ln (1-\mu \bar{\mu})\right\}\right) .
$$

It is straightforward to establish the following relation

$$
\partial_{z} \mu+\left(\partial_{\bar{z}}+\mu \partial_{z}\right) \ln \left(\partial_{z} w\right)=0
$$

with the help of which the term in $\ln \left(\partial_{z} w\right)$ may be eliminated to give (3.2). The derivation of (3.2) is complete. The formula (3.2) can be yet recast in a perhaps more suggestive form

$$
\tilde{\nabla}^{z} \varphi=\frac{e^{-2 \sigma}}{(1-\mu \bar{\mu})^{2-n}} \hat{\nabla}^{z} \hat{\varphi}
$$

where we have set $\hat{\varphi}=(1-\mu \bar{\mu})^{-n} \varphi$ and

$$
\hat{\nabla}^{z} \hat{\varphi}=g^{z \bar{z}}\left(\partial_{\bar{z}} \hat{\varphi}+\mu \partial_{z} \hat{\varphi}+n\left(\partial_{z} \mu\right) \hat{\varphi}\right) .
$$

The covariant derivative $\hat{\nabla}^{z} \hat{\varphi}$ now precisely coincides with the covariant derivative to first order in $\mu$, though (3.3) is now valid for finite deformations $\mu$. 


\section{Finite deformations via the stress tensor}

Using the theory and practice of finite deformations of complex structures developed in the preceding sections, we shall now apply these finite deformations to the standard worldsheet actions for superstring theory, namely for the scalar matter field $x^{\nu}$ and the spinor matter field $\psi^{\nu}$ in flat space-time, and for the ghost fields $b, c, \beta, \gamma$. (Actions for non-linear $\sigma$-models for string theory in curved space-time may be treated in an analogous manner.) We will recover the same actions that one would obtain by including a finite deformation of the worldsheet metric, as expected. We will then show that these finite deformations are fully accounted for by the inclusion of the usual stress tensors for these fields, provided the overall total central charge vanishes.

\subsection{Finite deformations of worldsheet actions}

The simplest case is the action for the scalar fields $x^{\nu}$. Denoting their deformations to fields in $T^{(0,0)}\left(\Sigma_{w}\right)$ by $\tilde{x}^{\nu}$, the scalar action is given by

$$
\tilde{I}_{x}=\frac{1}{4 \pi} \int_{\Sigma} d^{2} w \partial_{w} \tilde{x}^{\nu} \partial_{\bar{w}} \tilde{x}^{\nu}
$$

where $\nu$ is the 10-dimensional space-time index, contracted with the help of the flat Minkowski space-time metric by summation over repeated indices $\nu$. Using the first equation in (2.3) to recast the measure in terms of $z$, the second equation of (2.3) to recast the derivatives in terms of derivatives with respect to $z$, and denoting the corresponding scalar field in $T^{(0,0)}\left(\Sigma_{z}\right)$ by $x^{\nu}$, we obtain the following action

$$
\tilde{I}_{x}=\frac{1}{4 \pi} \int \frac{d^{2} z}{1-\mu \bar{\mu}}\left((1+\mu \bar{\mu}) \partial_{z} x^{\nu} \partial_{\bar{z}} x^{\nu}+\bar{\mu} \partial_{\bar{z}} x^{\nu} \partial_{\bar{z}} x^{\nu}+\mu \partial_{z} x^{\nu} \partial_{z} x^{\nu}\right) .
$$

Clearly, this action is not new; one arrives at the same expression for $\tilde{I}_{x}$ by considering the action for the scalar field $x^{\nu}$

$$
I_{x}=\frac{1}{8 \pi} \int_{\Sigma} d^{2} \xi \sqrt{g} g^{m n} \partial_{m} x^{\nu} \partial_{n} x^{\nu}
$$

in the presence of a general worldsheet metric $g_{m n}$ with $m, n=1,2$, for arbitrary coordinates $\xi^{1}, \xi^{2}$ on $\Sigma$. To recover $\tilde{I}_{x}$, it then suffices to choose the following parametrization

$$
d s^{2}=g_{m n} d \xi^{m} d \xi^{n}=2 e^{2 \sigma(z)}|d z-\mu(z) d \bar{z}|^{2} .
$$

The dependence on $\sigma(z)$ again drops out in view of the Weyl invariance of the action $I_{x}$.

The action for the spin $1 / 2$ field $\tilde{\psi}^{\nu}$ belonging to $T^{\left(\frac{1}{2}, 0\right)}\left(\Sigma_{w}\right)$ is given by

$$
\tilde{I}_{\psi}=-\frac{1}{4 \pi} \int_{\Sigma} d^{2} w \tilde{\psi}^{\nu} \partial_{\bar{w}} \tilde{\psi}^{\nu}
$$

In terms of the field $\psi^{\nu} \in T^{\left(\frac{1}{2}, 0\right)}\left(\Sigma_{z}\right)$, it is given by

$$
\tilde{I}_{\psi}=-\frac{1}{4 \pi} \int_{\Sigma} \frac{d^{2} z}{1-\mu \bar{\mu}}\left(\psi^{\nu} \partial_{\bar{z}} \psi^{\nu}+\mu \psi^{\nu} \partial_{z} \psi^{\nu}\right)
$$

The contribution arising from terms of the form $\psi^{\nu} \psi^{\nu}$ actually vanishes in view of the Grassmann nature of the field $\psi^{\nu}$. The resulting action in turn may be obtained alternatively by starting from an action for $\psi^{\nu}$ for a general metric parametrized by (4.1). 
Finally, we consider the theory of pairs of fields $b(z), c(z)$ belonging to $T^{n, 0}\left(\Sigma_{z}\right)$ and $T^{1-n, 0}\left(\Sigma_{z}\right)$ respectively. Denote by $\tilde{b}(w)$ and $\tilde{c}(w)$ respectively their deformations to fields in $T^{n, 0}\left(\Sigma_{w}\right)$ and $T^{1-n, 0}\left(\Sigma_{w}\right)$. The action is given by

$$
\tilde{I}_{b c}=\frac{1}{2 \pi} \int d^{2} w \tilde{b} \partial_{\bar{w}} \tilde{c}
$$

In terms of the fields $b(z), c(z)$, the deformed action is given by

$$
\tilde{I}_{b c}=\frac{1}{2 \pi} \int_{\Sigma} \frac{d^{2} z}{1-\mu \bar{\mu}}\left(b \partial_{\bar{z}} c+\mu b \partial_{z} c-(n-1) b c \partial_{z} \mu+(n-1) b c\left(\partial_{\bar{z}}+\mu \partial_{z}\right) \ln (1-\mu \bar{\mu})\right) .
$$

The action for the superghosts $\beta, \gamma$ is obtained by replacing $b$ by $\beta$ and $c$ by $\gamma$ and setting $n=\frac{3}{2}$. For the case of $n=\frac{1}{2}$ the $b$ and $c$ fields have the same weight. The case of the $\psi$ field treated earlier then corresponds to identifying $b$ and $c$ which is possible only at $n=\frac{1}{2}$.

Putting together the cases of $n=0, n=\frac{1}{2}, n=2$, and $n=\frac{3}{2}$, we will obtain the expression to all orders in the deformation $\mu$ for the worldsheet action for the RNS string, at vanishing worldsheet gravitino field. It is well-known how to include the latter contribution.

\subsection{Chiral splitting}

Each classical action, $\tilde{I}_{x}, \tilde{I}_{\psi}, \tilde{I}_{b c}$, and $\tilde{I}_{\beta \gamma}$, depends on both $\mu$ and $\bar{\mu}$. It is a basic principle of two dimensional conformal field theories that, in the deformation of their correlation functions, all terms involving $\mu \bar{\mu}$ should appear with a coefficient proportional to their central charges. Indeed, each quantum partition function depends on both $\mu$ and $\bar{\mu}$, but it is well-known from Belavin and Knizhnik [1] that this mixed dependence is proportional to the central charge of each field. The quantum partition function of the combined fields $x^{\nu}, \psi^{\nu}, b, c, \bar{b}, \bar{c}, \beta, \gamma, \bar{\beta}, \bar{\gamma}$ has vanishing central charge and, properly normalized, is the absolute value square of a chiral partition function, which only depends on $\mu$ and not on $\bar{\mu}$. A generalization of this result to the case of the full worldsheet supergravity including the dependence on the Beltrami differential $\mu$ and the worldsheet gravitino field $\chi$ was proven in [3].

The above result may be further generalized to the case of superstring amplitudes with $N$ external string states represented by vertex operator insertions on the worldsheet, and gives rise to the chiral splitting theorem, proven in [5] for strings propagating in flat Minkowski spacetime or on a flat toroidal compactification thereof. In brief, the theorem states that a string amplitude in which left and right movers are complex conjugates of one another, with equal fixed internal loop momenta $p$ and equal fixed spin structure $\delta$, is the absolute value square of a chiral string amplitude which depends only on $\mu$ and $\chi$ but not on their complex conjugates. The physical Type II string amplitudes are then obtained by pairing left and right chiral amplitudes at the same internal loop momenta $p$, but different spin structures $\delta_{L}$ and $\delta_{R}$ which are to be summed over independently in accord with the GSO projection. For the heterotic strings, the prescription is analogous with the right moving chiral amplitude replaced by the chiral half of the bosonic string compactified on a 16-dimensional torus. In summary, the basic building blocks in the perturbation theory of all closed oriented string theories are the chiral string amplitudes which depend on $\mu$ but are independent of $\bar{\mu}$.

\subsection{Finite deformations via the stress tensor}

Thus, in a theory with total central charge 0, as in superstring perturbation theory, we can just drop all the dependence involving $\mu \bar{\mu}$. In view of all the formulas derived in the previous sections for finite deformations of complex structures, we find the remarkable fact that the covariant derivatives, the actions, and the stress tensors are all given by the same formulas as 
for infinitesimal deformations, but with the property that the formulas are valid to all orders of expansion in $\mu$. The corresponding effective chiral action for the chiral parts of the fields (denoted $x_{+}^{\nu}$ and $\psi_{+}^{\nu}$ for the matter RNS fields) of the superstring thus takes the form

$$
I=\frac{1}{2 \pi} \int_{\Sigma} d^{2} z\left(\frac{1}{2} \partial_{\bar{z}} x_{+}^{\nu} \partial_{z} x_{+}^{\nu}-\frac{1}{2} \psi_{+}^{\nu} \partial_{\bar{z}} \psi_{+}^{\nu}+b \partial_{\bar{z}} c+\beta \partial_{\bar{z}} \gamma-\mu T-\chi S\right),
$$

where $T$ is the worldsheet stress tensor obtained from the expressions of the actions given above, and truncated by setting $\bar{\mu}=0$. Also, $S$ is the worldsheet supercurrent, whose effects have been included for completeness, but without derivation. This formula was the starting point for the construction of two-loop amplitudes with NS vertex operators in $[7,8]$, where the formula was needed only to first order in $\mu$, but here it is established to all orders in $\mu$. We also refer to [16, Section 3.5] for a helpful treatment of deformations of supercomplex structures by fields.

Collecting all terms for the stress tensor, we find the well-known form [12]

$$
T=-\frac{1}{2} \partial_{z} x_{+}^{\nu} \partial_{z} x_{+}^{\nu}+\frac{1}{2} \psi^{\nu} \partial_{z} \psi_{+}^{\nu}-b \partial_{z} c-\partial_{z}(b c)-\beta \partial_{z} \gamma-\frac{1}{2} \partial_{z}(\beta \gamma) .
$$

The expression for the supercurrent is similarly given by [12]

$$
S=-\frac{1}{2} \psi_{+}^{\nu} \partial_{z} x_{+}^{\nu}+\frac{1}{2} b \gamma-\frac{1}{2} \beta \partial_{z} c-\partial_{z}(\beta c) .
$$

Note that neither $T$ nor $S$ involves any dependence on $\mu$, a key ingredient in the conclusion that the formulas for deformations by $\mu$ customarily viewed as valid only to first order in $\mu$, are actually valid to all orders in $\mu$. Also note that $\chi$ is independent of $\mu$, so that when the above action is used to higher order in $\mu$, there is no need to deform the complex structure in which $\chi$ was originally defined: all such deformations will effectively be taken care of by the insertion of the stress tensor term $\mu T$.

\subsection{Finite deformations of Green functions}

The chiral splitting principle brings about an enormous simplification. We shall now test this explicitly by showing that the Green functions for the scalar field $x_{+}^{\nu}$, for the spinor field $\psi_{+}^{\nu}$, and for the ghost systems $b, c$ and $\beta, \gamma$ are precisely given by the correlators for these fields in the presence of their respective stress tensor, and this to all orders in $\mu$. For the sake of clarity we shall treat each system of fields separately. Our main concern is with higher loop superstring amplitudes for closed oriented superstrings, so we shall discuss the case when $\Sigma$ is a closed oriented Riemann surface of genus $h \geq 2$. For the case of the sphere $h=0$, the complex structure is unique, while for the torus $h=1$ the situation is well-known.

\subsection{The $b, c$ system}

The $b, c$ system has anti-commuting fields $b, c$ and will be considered here for arbitrary $n \geq \frac{3}{2}$ (we shall discuss the cases of $n=0, \frac{1}{2}, 1$ separately below). We shall show that the Green function for the Cauchy-Riemann operator $\hat{\nabla}_{\mu}^{z}$ is identical to the correlator for the $b, c$ system in the presence of the stress tensor insertion $\mu T$, to all orders in $\mu$. Recall that the covariant derivative $\hat{\nabla}_{\mu}^{z}$, defined earlier in (3.3), acting on a tensor field of weight $(n, 0)$, is given by

$$
\hat{\nabla}_{\mu}^{z}=\frac{1}{\sqrt{\tilde{g}}}\left(\partial_{\bar{z}}+\mu \partial_{z}+n\left(\partial_{z} \mu\right)\right)
$$

For the case considered here, namely $n \geq 3 / 2$, the kernel of the adjoint of $\hat{\nabla}_{\mu}^{z}$ vanishes, and the Green function is simply defined by

$$
\tilde{\nabla}_{\mu}^{z} G_{\mu}\left(z, z^{\prime}\right)=2 \pi \delta_{\tilde{g}}\left(z, z^{\prime}\right) \text {. }
$$


By conformal invariance, all factors of $\tilde{g}$ cancel on both sides above so that the equations which define the Green functions for $\mu$ and for $\mu=0$ are given by

$$
\begin{aligned}
& \left(\partial_{\bar{z}}+\mu \partial_{z}+n\left(\partial_{z} \mu\right)\right) G_{\mu}\left(z, z^{\prime}\right)=2 \pi \delta\left(z-z^{\prime}\right) \\
& \partial_{\bar{z}} G_{0}\left(z, z^{\prime}\right)=2 \pi \delta\left(z-z^{\prime}\right) .
\end{aligned}
$$

To calculate $G_{\mu}$ in terms of $G_{0}$ to all orders in $\mu$, we combine the above equations

$$
\partial_{\bar{z}} G_{\mu}\left(z, z^{\prime}\right)=\partial_{\bar{z}} G_{0}\left(z, z^{\prime}\right)-\left(\mu \partial_{z}+n\left(\partial_{z} \mu\right)\right) G_{\mu}\left(z, z^{\prime}\right)
$$

which may be integrated as follows

$$
G_{\mu}\left(z, z^{\prime}\right)=G_{0}\left(z, z^{\prime}\right)-\frac{1}{2 \pi} \int d^{2} v G_{0}(z, v)\left(\mu \partial_{v}+n\left(\partial_{v} \mu\right)\right) G_{\mu}\left(v, z^{\prime}\right) .
$$

In general, this integration will allow for an additional contribution which is a holomorphic form of weight $(n, 0)$ in $z$, since the $\partial_{\bar{z}}$ operator on $(n, 0)$-forms has a non-trivial kernel. This arbitrariness may be fixed, for example, by insisting that the free zeros of $G_{\mu}$ in its first argument, $z$, be independent of $\mu$, and coincide with the free zeros of $G_{0}$. Denoting these free zeros by $z_{a}$ with $a=1, \cdots, \Upsilon(n)$ where $\Upsilon(n)=(2 n-1)(h-1)$, for the case $h \geq 2$ of interest here, we are led to require

$$
G_{\mu}\left(z_{a}, z^{\prime}\right)=0, \quad a=1, \ldots, \Upsilon(n)
$$

for all points $z^{\prime}$, arbitrary $\mu$ including 0 , and $z_{a}$ independent of $\mu$. This requirement is natural in superstring perturbation theory. The Green function $G_{\mu}$ may be obtained to arbitrary order in $\mu$ by iterating the integral equation, and we get schematically

$$
G_{\mu}=G_{0}+G_{0} M G_{0}+G_{0} M G_{0} M G_{0}+\cdots,
$$

where $M$ is the operator $-\left(\mu \partial_{v}+n\left(\partial_{v} \mu\right)\right) /(2 \pi)$.

\subsubsection{Green function by deformed correlator}

We shall now prove that the Green function $G_{\mu}$ is alternatively given in terms of the chiral deformed correlator for the $b c$ system by

$$
G_{\mu}\left(z, z^{\prime}\right)=\left\langle b(z) c\left(z^{\prime}\right)\right\rangle_{\mu}
$$

where the correlator $\left\langle b(z) c\left(z^{\prime}\right)\right\rangle_{\mu}$ is defined as follows

$$
\left.\left\langle b(z) c\left(z^{\prime}\right)\right\rangle_{\mu}=\frac{1}{Z_{b c}}\left\langle b(z) c\left(z^{\prime}\right) \prod_{a=1}^{\Upsilon(n)} b\left(z_{a}\right) \exp \left\{\frac{1}{2 \pi} \int d^{2} v \mu(v) T_{b c}(v)\right)\right\}\right\rangle_{0}
$$

and the stress tensor is given by

$$
T_{b c}=-b \partial_{z} c-(n-1) \partial_{z}(b c) .
$$

The normalization factor $Z_{b c}$ is defined by omitting the insertions $b(z) c\left(z^{\prime}\right)$, and is given by

$$
\left.Z_{b c}=\left\langle\prod_{a=1}^{\Upsilon(n)} b\left(z_{a}\right) \exp \left\{\frac{1}{2 \pi} \int d^{2} v \mu(v) T_{b c}(v)\right)\right\}\right\rangle_{0}
$$


The expectation value $\langle\cdots\rangle_{0}$ is with respect to the action for the $b c$ system for vanishing $\mu$. In particular, $\left\langle b(z) c\left(z^{\prime}\right)\right\rangle_{0}$ equals $G_{0}\left(z, z^{\prime}\right)$ and has a pole of unit residue at $z=z^{\prime}$

$$
\left\langle b(z) c\left(z^{\prime}\right)\right\rangle_{0}=G_{0}\left(z, z^{\prime}\right)=\frac{1}{z-z^{\prime}}+\mathcal{O}\left(\left(z-z^{\prime}\right)^{0}\right) .
$$

To prove the proposed equality (4.7), one may proceed either by showing that the above expression obeys the differential equation for $G_{\mu}$ in (4.3) as well as the normalization conditions (4.5), or alternatively that its expansion in powers of $\mu$ coincides with the one given in (4.6). It is instructive to give both proofs.

By construction, both $G_{\mu}\left(z, z^{\prime}\right)$ and $\left\langle b(z) c\left(z^{\prime}\right)\right\rangle_{\mu}$ are forms of weight $(n, 0)$ in $z$ and weight $(1-n, 0)$ in $z^{\prime}$, both vanish at $z=z_{a}$ for all $a=1, \ldots, \Upsilon(n)$, and both have a simple pole with unit residue in $z$ at $z=z^{\prime}$. Furthermore, the operator product of the fields $b$ and $T$ exhibits a universal pole governed by the dimension $n$ of $b$, namely,

$$
b(z) T_{b c}(v)=\frac{n b(v)}{(v-z)^{2}}+(n-1) \frac{\partial_{v} b(v)}{z-v}+\mathcal{O}\left((z-v)^{0}\right) .
$$

Upon integrating $T_{b c}$ against $\mu$, and applying the Cauchy-Riemann operator $\partial_{\bar{z}}$, we have

$$
\partial_{\bar{z}}\left(b(z) \int_{\Sigma} d^{2} v \mu(v) T_{b c}(v)\right)=-\mu(z) \partial_{z} b(z)-n\left(\partial_{z} \mu(z)\right) b(z) .
$$

As a result, one finds that $\left\langle b(z) c\left(z^{\prime}\right)\right\rangle_{\mu}$ obeys the first equation in (4.3). Since it also obeys the normalization conditions (4.5) this completes the proof of (4.7) to all orders in $\mu$.

Alternatively, one may compare the expansions of $G_{\mu}\left(z, z^{\prime}\right)$ and $\left\langle b(z) c\left(z^{\prime}\right)\right\rangle_{\mu}$ in powers of $\mu$ term by term. Concentrating, for example, on the term of first order in $\mu$, we have

$$
\left\langle b(z) c\left(z^{\prime}\right)\right\rangle_{\mu}=\left\langle b(z) c\left(z^{\prime}\right)\right\rangle_{0}+\frac{1}{2 \pi} \int d^{2} v \mu(v)\left\langle T_{b c}(v) b(z) c\left(z^{\prime}\right)\right\rangle_{0}+\mathcal{O}\left(\mu^{2}\right),
$$

where $T$ was given in (4.8). Using Wick contractions to carry out the correlator, we find

$$
\int d^{2} v \mu(v)\left\langle T_{b c}(v) b(z) c\left(z^{\prime}\right)\right\rangle_{0}=-\int_{\Sigma} d^{2} v G_{0}(z, v)\left(\mu(v) \partial_{v}+n \partial_{v} \mu(v)\right) G_{0}\left(v, z^{\prime}\right) .
$$

Comparison with (4.4) shows perfect agreement. Terms of higher order may be identified in an analogous manner.

\subsection{The $\beta, \gamma$ system}

The only difference between the $\beta, \gamma$ system and the $b, c$ system discussed above is that $\beta$ and $\gamma$ are commuting fields while $b, c$ were anti-commuting fields. The $\beta, \gamma$ system will be considered here for $n \geq \frac{3}{2}$. In particular, the Green function for the Cauchy-Riemann operator $\tilde{\nabla}_{\mu}^{z}$ is identical to the one for the $b, c$ system, and is therefore defined by the differential equation (4.3) along with the normalization conditions (4.5) for the appropriate value of $n$, namely $n=\frac{3}{2}$ in the case of the superstring superghost system. The result for the $\beta, \gamma$ system is as follows

$$
G_{\mu}\left(z, z^{\prime}\right)=-\left\langle\beta(z) \gamma\left(z^{\prime}\right)\right\rangle_{\mu},
$$

where the correlator $\left\langle\beta(z) \gamma\left(z^{\prime}\right)\right\rangle_{\mu}$ is defined as follows

$$
\left.\left\langle\beta(z) \gamma\left(z^{\prime}\right)\right\rangle_{\mu}=\frac{1}{Z_{\beta \gamma}}\left\langle\beta(z) \gamma\left(z^{\prime}\right) \prod_{a=1}^{\Upsilon(n)} \delta\left(\beta\left(z_{a}\right)\right) \exp \left\{\frac{1}{2 \pi} \int d^{2} v \mu(v) T_{\beta \gamma}(v)\right)\right\}\right\rangle_{0}
$$


and the stress tensor is given by

$$
T_{\beta \gamma}=-\beta \partial_{z} \gamma-(n-1) \partial_{z}(\beta \gamma)
$$

The normalization factor $Z_{\beta \gamma}$ is defined by omitting the insertions $\beta(z) \gamma\left(z^{\prime}\right)$, and given by

$$
\left.Z_{\beta \gamma}=\left\langle\prod_{a=1}^{\Upsilon(n)} \delta\left(\beta\left(z_{a}\right)\right) \exp \left\{\frac{1}{2 \pi} \int d^{2} v \mu(v) T_{\beta \gamma}(v)\right)\right\}\right\rangle_{0} .
$$

Note that the sign difference in (4.11) constitutes a crucial difference with the $b, c$ system. In particular, the correlator at $\mu=0$ has a single pole at $z=z^{\prime}$ with residue -1 , as given by (4.9). For useful treatments of the $\beta, \gamma$ correlators, we refer the reader to $[13,15]$ and $[17$, Section 10].

\subsection{The spinor field $\psi_{+}^{\mu}$}

The spinor field $\psi$ (we shall drop the Lorentz superscript $\nu$ and the chirality subscript + in this section), may be viewed as the special case of the $b, c$ system with $n=\frac{1}{2}$ and the fields $b$ and $c$ identified. The Green function is now referred to as the Szegö kernel. The Cauchy-Riemann operator $\tilde{\nabla}_{\mu}^{z}$ is given by (4.2) with $n=\frac{1}{2}$. For even spin structures, the kernel of $\tilde{\nabla}_{\mu}^{z}$ and the kernel of its adjoint operator are both null, generically on moduli space. For odd spin structure, however, both operators have a one-dimensional kernel, again generically on moduli space.

For even spin structure, the Szegö kernel will be denoted by $S_{\mu}\left(z, z^{\prime}\right)$. It is odd under the interchange of $z$ and $z^{\prime}$ and satisfies the differential equation

$$
\begin{aligned}
& \left(\partial_{\bar{z}}+\mu \partial_{z}+\frac{1}{2}\left(\partial_{z} \mu\right)\right) S_{\mu}\left(z, z^{\prime}\right)=2 \pi \delta\left(z-z^{\prime}\right) \\
& \partial_{\bar{z}} S_{0}\left(z, z^{\prime}\right)=2 \pi \delta\left(z-z^{\prime}\right)
\end{aligned}
$$

away from non-generic points on moduli space where the kernel and co-kernel of $\tilde{\nabla}_{\mu}^{z}$ are nontrivial. The expression for $S_{\mu}\left(z, z^{\prime}\right)$ in terms of $S_{0}$ and $\mu$ to all orders in $\mu$ is given by an immediate adaptation of (4.4) to this case, namely by iterating to an arbitrary order in $\mu$ the integral equation

$$
S_{\mu}\left(z, z^{\prime}\right)=S_{0}\left(z, z^{\prime}\right)-\frac{1}{2 \pi} \int d^{2} v S_{0}(z, v)\left(\mu \partial_{v}+\frac{1}{2}\left(\partial_{v} \mu\right)\right) S_{\mu}\left(v, z^{\prime}\right) .
$$

The deformed Szegö kernel is then related to the deformed correlator of the field $\psi$ by

$$
S_{\mu}\left(z, z^{\prime}\right)=-\left\langle\psi(z) \psi\left(z^{\prime}\right)\right\rangle_{\mu},
$$

where the correlator $\left\langle\psi(z) \psi\left(z^{\prime}\right)\right\rangle_{\mu}$ is defined as follows,

$$
\left.\left\langle\psi(z) \psi\left(z^{\prime}\right)\right\rangle_{\mu}=\frac{1}{Z_{\psi}}\left\langle\psi(z) \psi\left(z^{\prime}\right) \exp \left\{\frac{1}{2 \pi} \int d^{2} v \mu(v) T_{\psi}(v)\right)\right\}\right\rangle_{0}
$$

and the stress tensor is given by

$$
T_{\psi}=\frac{1}{2} \psi \partial_{z} \psi
$$

The normalization factor $Z_{\psi}$ is defined by omitting the insertions $\psi(z) \psi\left(z^{\prime}\right)$, and is given by

$$
\left.Z_{\psi}=\left\langle\exp \left\{\frac{1}{2 \pi} \int d^{2} v \mu(v) T_{\psi}(v)\right)\right\}\right\rangle_{0} .
$$

The expectation value $\langle\cdots\rangle_{0}$ is with respect to the action for the $\psi$ system for vanishing $\mu$. 
For odd spin structure, generically on moduli space, the Cauchy-Riemann operator $\tilde{\nabla}_{\mu}^{z}$, as well as its adjoint, have one zero mode which we shall denote by $h_{\mu}(z)$, and which satisfies

$$
\partial_{\bar{z}} h_{\mu}+\mu \partial_{z} h_{\mu}+\frac{1}{2}\left(\partial_{z} \mu\right) h_{\mu}=0
$$

Due to the presence of this zero mode, there is no unique way of defining the Szegö kernel, and it is generally not odd under the interchange of $z, z^{\prime}$. To make the connection with correlators and chiral splitting, it will be convenient to consider a pair of chiral fermions $\psi^{1}, \psi^{2}$, and denote their complex linear combinations as follows, $\psi=\left(\psi^{1}+i \psi^{2}\right) / \sqrt{2}$ and $\bar{\psi}=\left(\psi^{1}-i \psi^{2}\right) / \sqrt{2}$. One may think of $\psi$ and $\bar{\psi}$ as the fields $b$ and $c$ of the $n=\frac{1}{2}$ system without having made the identification of the field $b$ with the field $c$. Introducing on $\Sigma$ an arbitrary point $\zeta$ at which we have $h_{\mu}(\zeta) \neq 0$, the Szegö kernel $S_{\mu}\left(z, z^{\prime} ; \zeta\right)$ may be defined as satisfying the following differential equation

$$
\left(\partial_{\bar{z}}+\mu \partial_{z}+\frac{1}{2}\left(\partial_{z} \mu\right)\right) S_{\mu}\left(z, z^{\prime} ; \zeta\right)=2 \pi \delta\left(z-z^{\prime}\right)-2 \pi \delta(z-\zeta) \frac{h_{\mu}\left(z^{\prime}\right)}{h_{\mu}(\zeta)}
$$

Integration of both sides against $h_{\mu}(z)$ vanishes, and this is the rationale for the choice of the subtraction term on the right hand side. When $z^{\prime}=\zeta$, the right hand side vanishes, so that $\left.S_{\mu}\left(z, z^{\prime} ; \zeta\right)\right|_{z^{\prime}=\zeta}$ must be proportional to $h_{\mu}(z)$. Therefore, it is natural to set the Szegö kernel to zero at $z^{\prime}=\zeta$. This condition is analogous to (4.5) for the $b, c$-system, and defines the Szegö kernel uniquely. We shall denote the unique free zero in $z$ of $S_{\mu}\left(z, z^{\prime} ; \zeta\right)$ by $\zeta^{\prime}$.

We are led to proposing the following identification of the Szegö and correlator

$$
S_{\mu}\left(z, z^{\prime} ; \zeta\right)=-\left\langle\psi(z) \bar{\psi}\left(z^{\prime}\right)\right\rangle_{\mu}
$$

where the correlator is given by

$$
\left.\left\langle\psi(z) \bar{\psi}\left(z^{\prime}\right)\right\rangle_{\mu}=\frac{1}{Z_{\psi \bar{\psi}}}\left\langle\psi(z) \bar{\psi}\left(z^{\prime}\right) \psi\left(\zeta^{\prime}\right) \bar{\psi}(\zeta) \exp \left\{\frac{1}{2 \pi} \int d^{2} v \mu(v) T_{\psi \bar{\psi}}(v)\right)\right\}\right\rangle_{0}
$$

and the stress tensor is given by

$$
T_{\psi \bar{\psi}}=-\frac{1}{2} \psi \partial_{z} \bar{\psi}+\frac{1}{2} \partial_{z} \psi \bar{\psi}
$$

The normalization factor $Z_{\psi \bar{\psi}}$ is defined by omitting the insertions $\psi(z) \bar{\psi}\left(z^{\prime}\right)$, and given by

$$
\left.Z_{\psi \bar{\psi}}=\left\langle\psi\left(\zeta^{\prime}\right) \bar{\psi}(\zeta) \exp \left\{\frac{1}{2 \pi} \int d^{2} v \mu(v) T_{\psi \bar{\psi}}(v)\right)\right\}\right\rangle_{0}
$$

The proposed relation (4.14) may be proven by applying the operator $\partial_{\bar{z}}+\mu \partial_{z}+\frac{1}{2}\left(\partial_{z} \mu\right)$ to the correlator (4.15) and using the OPE of $T_{\psi \bar{\psi}}$ with $\psi$. The normalization factor $Z_{\psi \bar{\psi}}$ guarantees that the first $\delta$-function on the right side of (4.13) arises with the proper factor of $2 \pi$. The factor of the second term on the right side of (4.13) arises with the help of the following relation

$$
\left.\frac{h_{\mu}\left(z^{\prime}\right)}{h_{\mu}(\zeta)}=\frac{1}{Z_{\psi \bar{\psi}}}\left\langle\psi\left(\zeta^{\prime}\right) \bar{\psi}\left(z^{\prime}\right) \exp \left\{\frac{1}{2 \pi} \int d^{2} v \mu(v) T_{\psi \bar{\psi}}(v)\right)\right\}\right\rangle_{0},
$$

which is easily proven by showing that the right hand side satisfies (4.12) as a function of $z^{\prime}$, and is equal to 1 at the point $z^{\prime}=\zeta$. 


\subsection{The scalar field $x_{+}^{\nu}$}

The scalar field $x$ (we shall omit the superscript $\nu$ and the subscript + in this section) is not properly a conformal field due to the presence of the constant zero mode, and it is preferable to work with its derivative $\partial_{z} x$ which is a conformal field of type $(1,0)$. The corresponding Green function is the third Abelian differential of the second kind, $\omega\left(z, z^{\prime}\right)=\partial_{z} \partial_{z^{\prime}} \ln E\left(z, z^{\prime}\right)$ for vanishing $\mu$, and generalizes as follows to the case of arbitrary $\mu$

$$
\partial_{\bar{z}} \omega_{\mu}\left(z, z^{\prime}\right)+\partial_{z}\left(\mu(z) \omega_{\mu}\left(z, z^{\prime}\right)\right)=2 \pi \partial_{z^{\prime}} \delta\left(z-z^{\prime}\right) .
$$

Note that both sides properly integrate to zero against a constant. The identification with the chiral correlator of the field $x$ is as follows

$$
\omega_{\mu}\left(z, z^{\prime}\right)=-\left\langle\partial_{z} x\left(z^{\prime}\right) \partial_{z} x\left(z^{\prime}\right)\right\rangle_{\mu}
$$

which is consistent with the well-known result at $\mu=0$ given by

$$
\left\langle x(z) x\left(z^{\prime}\right)\right\rangle_{0}=-\ln E\left(z, z^{\prime}\right)
$$

and its derivatives in $z$ and $z^{\prime}$. The correlator is defined as follows

$$
\left.\left\langle\partial_{z} x\left(z^{\prime}\right) \partial_{z} x\left(z^{\prime}\right)\right\rangle_{\mu}=\frac{1}{Z_{x}}\left\langle\partial_{z} x(z) \partial_{z}\left(z^{\prime}\right) \exp \left\{\frac{1}{2 \pi} \int d^{2} v \mu(v) T_{x}(v)\right)\right\}\right\rangle_{0} .
$$

The stress tensor is given by

$$
T_{x}=-\frac{1}{2} \partial_{z} x \partial_{z} x
$$

and the normalization factor is given by

$$
\left.Z_{x}=\left\langle\exp \left\{\frac{1}{2 \pi} \int d^{2} v \mu(v) T_{x}(v)\right)\right\}\right\rangle_{0} .
$$

In both correlators, only the chiral part of the field enters. This completes our derivation of the deformation to all orders in $\mu$ of every conformal field theory needed in the critical superstring in flat Minkowski space-time, and toroidal compactifications thereof.

\section{Acknowledgements}

We would like to acknowledge the organizers, Decio Levi, Willard Miller, Yvan Saint-Aubin, and Pavel Winternitz for inviting us to the enjoyable conference and celebration in honor of Luc Vinet at the Centre de Recherches Mathématiques. We would also like to thank the referees for their very careful reading of the paper. This research was supported in part by the National Science Foundation grants PHY-13-13986 and DMS-12-66033.

\section{References}

[1] Belavin A.A., Knizhnik V.G., Algebraic geometry and the geometry of quantum strings, Phys. Lett. B 168 (1986), 201-206.

[2] D'Hoker E., Phong D.H., The super period matrix with Ramond punctures in the supergravity formulation, arXiv:1501.02675.

[3] D’Hoker E., Phong D.H., Superholomorphic anomalies and supermoduli space, Nuclear Phys. B 292 (1987), 317-329. 
[4] D'Hoker E., Phong D.H., The geometry of string perturbation theory, Rev. Modern Phys. 60 (1988), 9171065.

[5] D'Hoker E., Phong D.H., Conformal scalar fields and chiral splitting on super Riemann surfaces, Comm. Math. Phys. 125 (1989), 469-513.

[6] D'Hoker E., Phong D.H., Two-loop superstrings. I. Main formulas, Phys. Lett. B 529 (2002), 241-255, hep-th/0110247.

[7] D'Hoker E., Phong D.H., Two-loop superstrings. II. The chiral measure on moduli space, Nuclear Phys. B 636 (2002), 3-60, hep-th/0110283.

[8] D'Hoker E., Phong D.H., Two-loop superstrings. V. Gauge slice independence of the $N$-point function, Nuclear Phys. B $\mathbf{7 1 5}$ (2005), 91-119, hep-th/0501196.

[9] D'Hoker E., Phong D.H., Two-loop superstrings. VI. Nonrenormalization theorems and the 4-point function, Nuclear Phys. B $\mathbf{7 1 5}$ (2005), 3-90, hep-th/0501197.

[10] D'Hoker E., Phong D.H., Lectures on two-loop superstrings, in Superstring Theory, Editors K. Liu, S.-T. Yau, C. Zhu, Advanced Lectures in Mathematics, Vol. 1, International Press, Boston, 2008, 85-123, hep-th/0211111.

[11] Friedan D., Introduction to Polyakov's string theory, in Recent Advances in Field Theory and Statistical Mechanics (Les Houches 1982), Editors J.B. Zuber, R. Stora, North-Holland Publishing Co., Amsterdam, 1984, 839-867.

[12] Friedan D., Martinec E., Shenker S., Conformal invariance, supersymmetry and string theory, Nuclear Phys. B 271 (1986), 93-165.

[13] Lechtenfeld O., Superconformal ghost correlations on Riemann surfaces, Phys. Lett. B 232 (1989), $193-198$.

[14] Royden H.L., The variation of harmonic differentials and their periods, in Complex Analysis, Birkhäuser, Basel, 1988, 211-223.

[15] Verlinde E., Verlinde H., Chiral bosonization, determinants and the string partition function, Nuclear Phys. B 288 (1987), 357-396.

[16] Witten E., Notes on super Riemann surfaces and their moduli, arXiv:1209.2459.

[17] Witten E., Superstring perturbation theory revisited, arXiv:1209.5461.

[18] Witten E., The super period matrix with Ramond punctures, J. Geom. Phys. 92 (2015), 210-239, arXiv:1501.0249.

[19] Yamada A., Precise variational formulas for abelian differentials, Kodai Math. J. 3 (1980), 114-143. 